\title{
ASPECTS OF THE DYNAMICS UNDERLYING SOLAR AND STELLAR DYNAMO MODELS
}

\author{
REZA TAVAKOL, EURICO COVAS \\ Astronomy Unit \\ School of Mathematical Sciences \\ Queen Mary \& Westfield College \\ Mile End Road \\ London E1 $4 N S$, UK \\ AND \\ DAVID MOSS \\ Department of Mathematics \\ The University \\ Manchester M13 9PL, UK
}

\begin{abstract}
Observations of the Sun and solar-type stars continue to reveal phenomena whose understanding is very likely to require a nonlinear framework. Here we shall concentrate on two such phenomena, namely the grand minima type behaviour observed in the Sun and solar-type stars and the recent dynamical variations of the differential rotation in the solar convection zone, deduced from the helioseismic observations, and discuss how their explanations have recently motivated the development/employment of novel ideas from nonlinear dynamics.
\end{abstract}

\section{Introduction}

An important characteristic of the Sun and solar-type stars is their variability over a very large range of time (and space) scales, including the intermediate time scales of $\sim 10^{0}-10^{4}$ years (Weiss 1990). Though ultimately spatiotemporal in character, these variabilities fall into two categories: (i) those whose main features can be explained in terms of temporal models and (ii) those which require a spatiotemporal approach for their explanations. 
Here we shall be focusing on two such forms of variability, namely the grand minima type episodes seen in in sunspot activity and the recently observed dynamical variations of the differential rotation in the solar convection zone.

Evidence for the presence of the grand-minima type behaviour comes from a variety of sources, including the studies of the historical records of the annual mean sunspot data since $1607 \mathrm{AD}$ which show the occurrence of epochs of suppressed sunspot activity, such as the Maunder minimum (Eddy 1976, Foukal 1990, Wilson 1994, Ribes \& Nesme-Ribes 1993, Hoyt \& Schatten 1996). Further research, employing ${ }^{14} C$ (Stuiver \& Quey 1980; Stuiver \& Braziunas 1988, 1989) and ${ }^{10} B$ (Beer et al. 1990, 1994a,b, Weiss \& Tobias 1997) as proxy indicators, has provided additional strong evidence that the occurrence of such epochs of reduced activity (referred to as grand minima) has persisted with irregular time intervals in the past. Further, they appear to be typical of solar-type stars (Baliunas et al. 1995).

Evidence for the variations in the dynamical behaviour of the differential rotation comes from recent analyses of the inversions of the helioseismic data from both the Michelson Doppler Imager (MDI) instrument on board the SOHO spacecraft and the Global Oscillation Network Group (GONG) project which provide evidence to show (i) that the previously observed surface torsional oscillations with periods of about 11 years (e.g. Howard \& LaBonte 1980; Snodgrass, Howard \& Webster 1985; Kosovichev \& Schou 1997; Schou et al. 1998) extend significantly downwards into the solar convective zone to depths of about 10 percent in radius (Howe et al. 2000a and Antia \& Basu 2000; see Covas et al. 2000a for a recent study), and (ii) that the dynamical regimes at the base of the convection zone may be different from those observed at the top, having either significantly shorter periods (Howe et al. 2000b) or non-periodic behaviour (Antia \& Basu 2000).

Our aim here is to briefly describe some recent attempts at understanding these phenomena within a nonlinear theoretical framework.

\section{Intermittency as a mechanism underlying grand minima type behaviour}

The seemingly irregular variations of the grand minima type in the sunspot activity are of interest for at least two reasons. Firstly, they are theoretically challenging, especially in view of the absence of any naturally occurring mechanisms in the Sun and solar-type stars with appropriate time scales (Gough 1990). Secondly, given their time scales, such variations can be of potential consequence for the occurrence of climatic variability on similar time scales (e.g. Friis-Christensen \& Lassen 1991, Beer at al. 1994b, Lean 1994, Stuiver, Grootes \& Braziunas 1995, Baliunas \& Soon 1995, Butler \& 
Johnston 1996, White et al. 1997).

These considerations have motivated considerable effort into trying to understand the mechanism(s) underlying such variations, employing a variety of approaches (e.g. Weiss et al. 1984; Sokoloff and Nesme-Ribes 1994; Tobias et al. 1995; Knobloch and Landsberg 1996; Schmitt et al. 1996, Knobloch et al. 1998; Tobias 1998a,b; Tobias et al. 1999).

In principle, there are essentially two frameworks within which such variabilities could be studied: stochastic and deterministic. In practice, however, it is difficult to distinguish between these two frameworks (Weiss 1990). Here we shall concentrate on the deterministic approach and recall that whatever the relevant framework may turn out to be, the deterministic components will still be present and are likely to play an important role.

Within this framework, a number of approaches have been adopted. Among these is the employment of low dimensional ODE models that are obtained using the Normal Form approach (Spiegel 1994, Tobias et al. 1995, Knobloch et al. 1996). These have led to robust modes of behaviour which are of potential importance in accounting for certain aspects of solar variability of the grand minima type, such as various forms of amplitude modulation of the magnetic field energy.

An alternative way to understand this type of variability in the sunspot record is to postulate that this type of behaviour is caused by some form of dynamical intermittency, an idea that in various forms goes back at least to the late 1970s (e.g. Tavakol 1978, Ruzmaikin 1981, Zeldovich et al. 1983, Weiss et al. 1984, Spiegel 1985, Feudel et al. 1993, Schmitt et al. 1996, Brooke 1997, Tworkowski et al. 1998).

Much effort has gone into producing evidence for the occurrence of various forms of dynamical intermittency in both truncated and PDE meanfield dynamo models. Given the large number of intermittency types that dynamical systems theory has produced and bearing in mind the complexity of the full dynamo equations, a natural approach would be to single out the main generic ingredients of stellar dynamos and to study their dynamical consequences. For axisymmetric dynamo models, these ingredients consist of the presence of invariant subspaces, non-normal parameters and the non-skew property (see e.g. Ashwin et al. 1999 for definitions). The dynamics underlying such systems has recently been studied (Covas et al. 1997c, 1999b; Ashwin et al. 1999), leading to a number of novel phenomena, including a new type of intermittency, referred to as in-out intermittency (see also Brooke 1997).

The easiest way to characterise in-out intermittency is by contrasting it with on-off intermittency. Briefly, let $M_{I}$ be the invariant subspace and $A$ the attractor which exhibits either on-off or in-out intermittency. One then has on-off or in-out intermittencies, depending respectively upon whether 
the intersection $A_{0}=A \cap M_{I}$ is a minimal attractor or not. The name in-out is chosen to be indicative of the fact that in this case there can be different invariant sets in $A_{0}$ associated with the attraction and repulsion transverse to $A_{0}$. Another crucial difference between the two is that, as opposed to on-off intermittency, in the case of in-out intermittency the minimal attractors in the invariant subspaces do not necessarily need to be chaotic and hence the trajectories can (and often do) shadow a periodic orbit in the 'out' phases (see Ashwin et al. 1999 for details).

Given the genericity of the ingredients required for the presence of this type of intermittency, it is of interest to see if this type of behaviour can indeed occur in the context of dynamo models. Interestingly, this form of intermittency has been concretely shown to occur in a number of dynamo models, including both ODE (Covas et al. 1997c) and PDE (Covas et al. 2000c) mean field dynamo models. An example of such an occurrence is given in Fig. 1, where in-out intermittency occurs for an axisymmetric PDE mean-field dynamo model (See Covas et al. (2000d) for details).

We should add here that in addition to in-out intermittency, firm evidence has also been found for the occurrence of other forms of intermittency in mean field dynamo models. These include crisis (or attractor merging) intermittency, in both ODE (Covas \& Tavakol 1997) and PDE (Covas \& Tavakol 1999) dynamo models, and Type I Intermittency, in both ODE (Covas et al. 1997c) and PDE models (Covas \& Tavakol 1999). We further note that on-off intermittency (Platt et al. 1993, see also Schmitt et al. 1996 and Ossendrijver 2000) is also likely to be relevant for such models, given their invariant subspaces.

The demonstration of the occurrence of various form of intermittency establishes an important element of the multiple intermittency hypothesis (Tavakol \& Covas 1999), according to which the grand minima type variability in solar-type stars may be understood in terms of one or a number of types of dynamical intermittency. What remains to be shown is whether these types of intermittency still persist in more realistic models and ultimately, through detailed comparison with observational and proxy data, whether they actually occur in the Sun and stars, and whether they can in fact account for the grand minima type behaviour.

The important point to be emphasised here is that in addition to theoretical predictions for these types of intermittency, their occurrence has been conclusively demonstrated not only in truncated dynamo models, but also in non-truncated PDE dynamo models (in three spatial dimensions, although so far restricted to axisymmetry, and time).

\section{Spatiotemporal bifurcations as a mechanism for different modes}



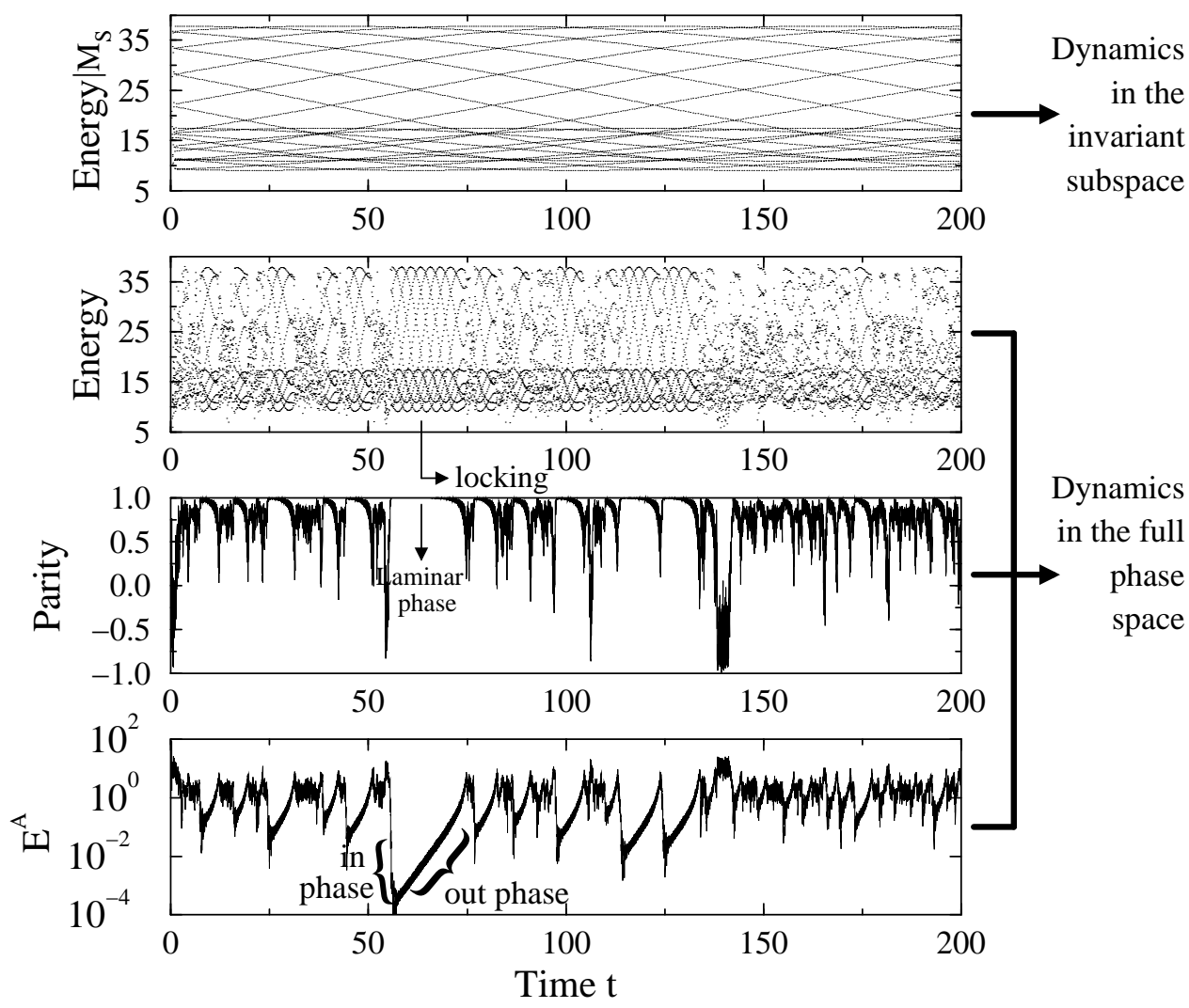

Figure 1. In-out intermittency in the axisymmetric PDE mean-field dynamo model. The parameters used were $r_{0}=0.4, C_{\alpha}=1.505, C_{\omega}=-10^{5}, f=0.7$, together with an algebraic form of $\alpha$. To enhance visually the periodic locking we have time sampled the series in the two upper panels. See Covas et al. (2000d) for details of the model.

\section{of behaviour in the solar convection zone}

An important outcome of the recent inversion of the helioseismic data has been to provide increasing evidence to show that the Sun is likely to be very active dynamically throughout the convection zone. To establish firmly the precise nature of these variations, future observations are clearly required. However, despite the error bars that such inversions are bound to entail, the results so far seem to point to the very interesting possibility that the variations in the differential rotation can have different periodicities/behaviours at different depths in the solar convection zone, having either markedly reduced periods (Howe et al. 2000b) or non-periodic behaviour (Antia \& Basu 2000) at the bottom of the convective zone.

There are in principle two ways to account for such behaviours. One 
could imagine different physical mechanisms at different physical locations in the convection zone, resulting in different dynamical behaviours in those locations. Alternatively, a dynamical mechanism could be sought that could produce such different behaviours at different spatial locations, without requiring different physical mechanisms at those locations.

As an example of the latter mechanism, spatiotemporal fragmentation/bifurcation has recently been proposed as a possible natural dynamical mechanism to account for such observed multi-mode behaviours in different parts of the solar convection zone (Covas et al. 2000b). In this way, dynamical regimes with different temporal behaviours can coexist at different spatial locations, for certain given values of the control parameters of the system. The important point here is that, in contrast to the usual temporal bifurcations, which result in identical temporal behaviour at each spatial point, and which require changes in parameters in order to be initiated, spatiotemporal bifurcations can result in different dynamical modes of behaviour at different locations without requiring changes in the control parameters. Also, importantly, the occurrence of such diverse modes of behaviour does not require different physical mechanisms at different locations.

Evidence for the occurrence of this mechanism was found in the context of a two dimensional axisymmetric mean field dynamo model operating in a spherical shell, with a semi-open outer boundary condition, in which the only nonlinearity is the action of the azimuthal component of the Lorentz force of the dynamo generated magnetic field on the solar angular velocity (Covas et al. 2000b). The zero order angular velocity was chosen to be consistent with the most recent helioseismological data (MDI). Subsequently it has been shown, through a detailed study (Covas et al. 2000c), that the occurrence of this type of behaviour does not depend upon the details of the model employed, thus providing strong evidence to support the idea that spatiotemporal fragmentation is likely to occur in general dynamo settings.

As an example of this type of spatiotemporal fragmentation/bifurcation, we have plotted in Fig. 2 the radial contours of the angular velocity residuals $\delta \Omega$, as a function of time for a cut at a fixed latitude. This demonstrates how, as a result of such spatiotemporal bifurcation, the period (as well ads the phase) of the angular velocity residuals vary going from the top to the bottom of the convection zone. The details of the model are given in Covas et al. (2000b,c).

To show the the presence of the spatiotemporal bifurcation more clearly, we also studied the bifurcation diagram by calculating the time between the minima of $\delta \Omega\left(r=0.66 R_{\odot}, \theta=30^{\circ}\right)$, the perturbation to the zero order rotation rate at a fixed latitude and radius, as a function of time.

Fig. 3 shows an example of such bifurcation, demonstrating clearly a 


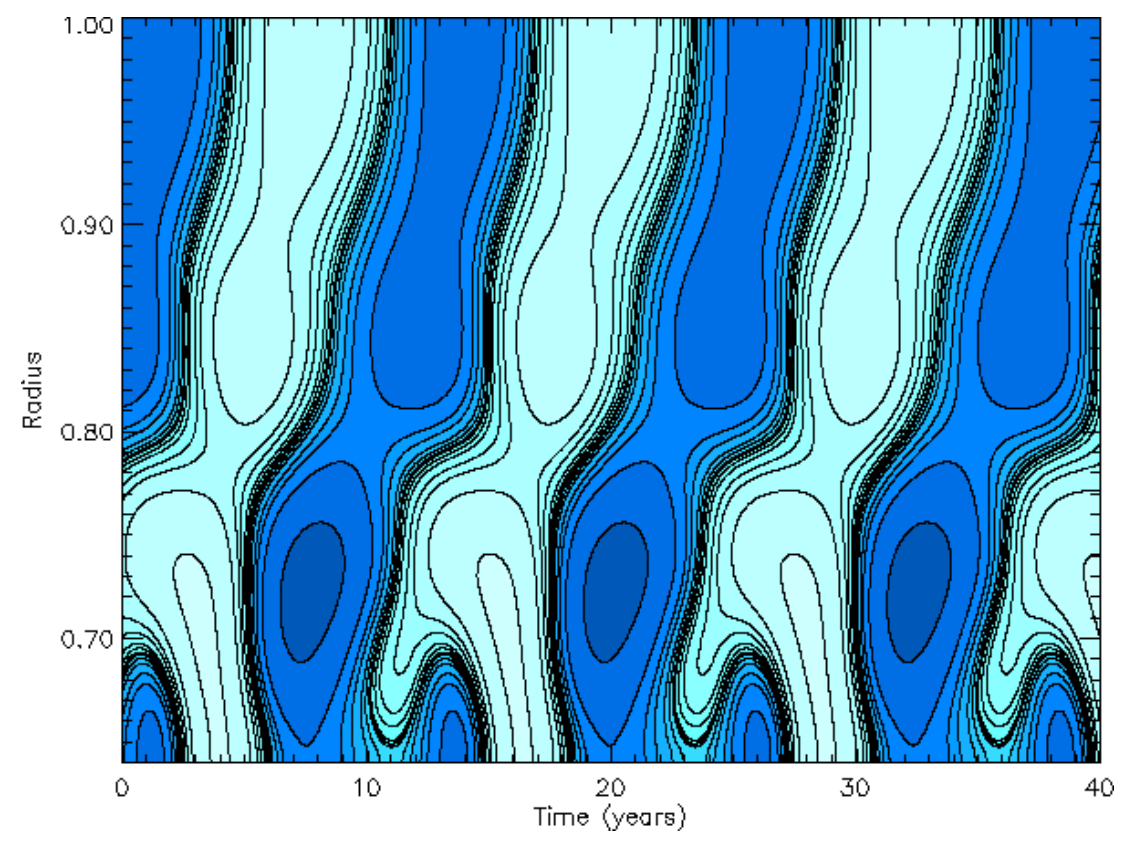

Figure 2. Radial contours of the angular velocity residuals $\delta \Omega$ as a function of time for a cut at latitude $30^{\circ}$. Parameter values are $R_{\alpha}=-11.0, P_{r}=0.9, R_{\omega}=44000$ for $\alpha(r, \theta)=\alpha_{r} \cos \theta \sin ^{2} \theta$. See Covas et al. (2000b) for the details of the model.

sudden bifurcation from a fundamental 11 year cycle to a cycle with two periods (whose sum is 11 years). Thus this mechanism is capable of producing different modes of behaviour at different locations, for given values of the control parameters of the system.

\section{Discussion}

We have briefly discussed a number of recent attempts at understanding two dynamical features observed in the Sun (and solar type stars), in terms of a number of novel concepts from nonlinear dynamics. A crucial feature of these types of explanation is that they are based on the nonlinear nature of the regimes under study, rather than on any specific physical mechanisms possessing the required time scale variabilities. This is important, particularly in view of the fact that much remains unknown about the underlying physics in these regimes.

We would like to thank Peter Ashwin, Axel Brandenburg, John Brooke, and Andrew Tworkowski for the work we have done together. EC is supported by a PPARC fellowship. 


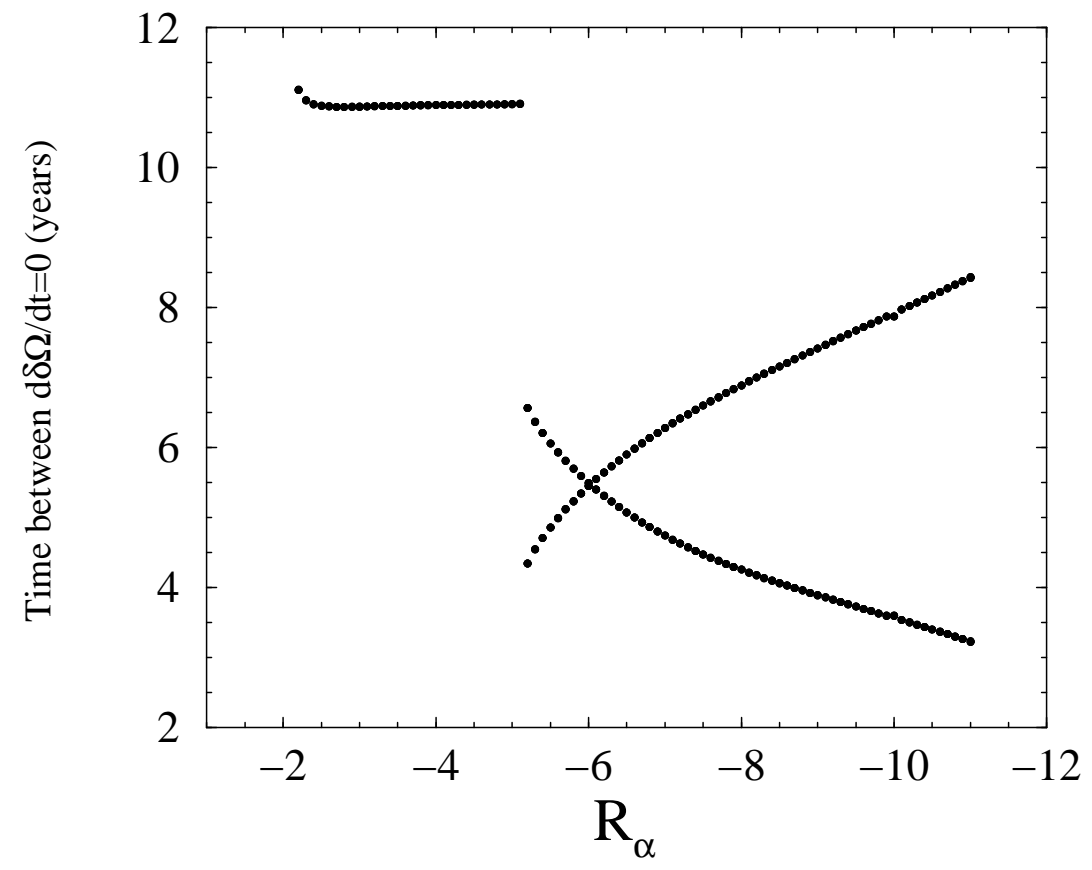

Figure 3. Bifurcation diagram showing the time between minima of the curve $\Omega(r, \theta$, time $)$. It shows clearly a sudden bifurcation from a fundamental 11 year cycle to a cycle with two timescales (with sum 11 years). Parameter values are $R_{\alpha}=-11.0$, $P_{r}=1.0, R_{\omega}=44000$ for a $\alpha(r, \theta)=\alpha_{r} \cos \theta \sin ^{2} \theta$. See Covas et al. (2000b) for the details of the model.

\section{References}

Antia, H. M., and Basu, S., 2000, ApJ, 541, 442

Ashwin, P., Covas, E. \& Tavakol, R., 1999, Nonlinearity, 9, 563.

Baliunas, S. L. \& Soon, W., 1995, Astrophy. J., 450, 896.

Baliunas, S. L., et al., 1995, Astrophysical Journal, 438, 269.

Beer, J., et al., 1994, in The Sun as a Variable Star: Solar and Stellar Irradiance Variations, J. M. Pap, C. Fröhlich, H. S. Hudson and S. K. Solaski, eds., Cambridge University Press, Cambridge, 291.

Beer, J. et al., 1994b, in E. Nesme-Ribes (ed.), The Solar Engine and its Influence on Terrestial Atmosphere and Climate, Springer-Verlag, Berlin, p. 221.

Beer, J. et al., 1990, Nature, 347, 164.

Brooke, J. M., 1997, Europhysics Letters, 37, 3.

Butler, C. J. \& Johnston, D. J., 1996, J. Atmospheric Terrest. Phys., 58, 1657.

Covas, E. \& Tavakol, R., 1997, Phys. Rev. E, 55, 6641.

Covas, E., Ashwin, P. \& Tavakol, R., 1997b, Phys. Rev. E, 56, 6451.

Covas, E. \& Tavakol, R., 1998, Proceedings of the 5th International Workshop "Planetary and Cosmic Dynamos", Trest, Czech Republic, Studia Geophysica et Geodaetica, 42.

Covas, E. \& Tavakol, R., 1999, Phys. Rev. E, 60, 5435.

Covas, E., Tavakol, R., Tworkowski, A., Brandenburg, A., Brooke, J. M. \& Moss, D., 1999, AEGA, 345, 669. 
Covas E., Tavakol R., Moss D., Tworkowski A., 2000a, A\&A 360, L21.

Covas, E., Tavakol, R., Moss, D., 2000b, A\&A 363, L13, also available at http://www.eurico.web.com

Covas, E., Tavakol, R., Moss, D., 2000c, submitted to A\&A.

Covas, E., Tavakol, R., Ashwin, P., Tworkowski, A., Brooke, J. , 2000d, submitted to Chaos.

Eddy, J. A., 1976, Science, 192, 1189.

Feudel, W. Jansen, \& J. Kurths, 1993, Int. J. of Bifurcation and Chaos, 3, 131.

Foukal, P. V., 1990, Solar Astrophysics, Wiley Interscience, New York.

Friis-Christensen, E. \& Lassen, K., 1991, Science 254, 698.

Gough, D., 1990, Phil. Trans. R. Soc. Lond., A330, 627.

Howard, R., and LaBonte, B. J., 1980, ApJ, 239, L33.

Howe, R., et al., 2000a, ApJ Lett., 533, L163.

Howe, R., et al., 2000b, Science 287, 2456.

Hoyt, D. V. \& Schatten, K. H., 1996, Solar Phys., 165, 181.

L. L. Kitchatinov, 1987, Geophys. Astrophys. Fluid Dyn., 38, 273.

Knobloch, E., and Landsberg, A. S., 1996, Mon. Not. Royal Astron. Soc., 278, 294.

Knobloch, E., Tobias, S. M. \& Weiss, N. O., 1998, MNRAS, 297, 1123.

Kosovichev, A. G., and Schou, J., 1997, ApJ, 482, L207.

Lean, J., 1994, in E. Nesme-Ribes (ed.), The Solar Engine and its Influence on Terrestial Atmosphere and Climate, Springer-Verlag, Berlin, p. 163.

Ossendrijver, M. A. J. H., 2000, A\&A, 359, 364.

Platt, N., Spiegel, E., and Tresser, C., 1993, Phys. Rev. Lett., 70, 279.

Ribes, J. C. \& Nesme-Ribes, E., 1993, A\&JA, 276, 549.

Ruzmaikin, A. A., 1981, Comm. Astrophys., 9, 88.

Schmitt, D., Schüssler, M., and Ferriz-Mas, A., 1996, A\& A, 311, L1.

Sokoloff, D., and Nesme-Ribes, E., 1994, A\&GA, 288, 293.

Snodgrass, H. B., Howard, R. F., and Webster, L., 1985, Sol. Phys., 95, 221.

Spiegel, E., Platt, N. \& Tresser, C., 1993b, Geophys. and Astrophys. Fluid Dyn., 73, 146.

Spiegel, E.A. 1994, in Proctor M.R.E., Gilbert A.D., eds, Lectures on Solar and Planetary Dynamos, Cambridge Univ. Press, Cambridge.

Spiegel, in Chaos in Astrophysics, edited by J. R. Butcher, J. Perdang, \& E. A. Spiegel (Reidel, Dordrecht, 1985).

Stuiver, M., Grootes, P. M. \& Braziunas, T. F., 1995, Quarternary Res., 44, 341.

Stuiver, M. \& Braziunas, T. F., 1988, in F. R. Stephenson \& A. W. Wolfendale (eds.), Secular Solar and Geomagnetic Variations in the Last 10000 Years, Kluwer Academic Publishers, Dordrecht, Holland, p. 245.

Stuiver, M. \& Braziunas, T. F., 1989, Nature, 338, 405.

Stuiver, M. \& Quay, P. D., 1980, Science, 207, 19.

Tavakol, R., 1978, Nature, 276, 802.

Tavakol, R., and Covas, E., 1999, Astron. Soc. of the Pacific Conference Series, 178, 173.

Tobias, S. M., Weiss, N. O., and Kirk, V., 1995, Mon. Not. Royal Astron. Soc., 273, 1150.

Tobias, S. M., 1998a, Astron. Soc. of the Pacific Conference Series, 154, 1349.

Tobias, S. M., 1998b, Mon. Not. Royal Astron. Soc., 296, 653.

Tobias, S. M., Knobloch, E., and Weiss, N. O., 1999, Astron. Soc. of the Pacific Conference Series, 178, 185.

Tworkowski, A., Tavakol, R., Brandenburg, A., Brooke, J. M., Moss, D. \& Tuominen I., 1998, MNRAS, 296, 287.

Weiss, N. O., Cattaneo, F., Jones, C. A., 1984, Geophys. Astrophys. Fluid Dyn., 30, 305.

Weiss, N. O., in Lectures on Solar and Planetary Dynamos, edited by Proctor, M.R.E. and Gilbert, A.D., Cambridge University Press, Cambridge (1994).

Weiss, N. O. \& Tobias, S. M., 1997, in Solar and Heliospheric Plasma Physics, ed. G. M. Simnett, C. E. Alissandrakis \& L. Vlahos, 25, Springer, Berlin.

Weiss, N. O., 1990, Phil. Trans. R. Soc. Lond., A330, 617. 
White, W. B., Lean, J., Cayan, D. \& Dettinger, M. D., 1997, J. Geophys. Res., 102, 3255.

Wilson, P. R., 1994, Solar and Stellar Activity Cycles, Cambridge University Press, Cambridge.

Zeldovich, Ya. B., Ruzmaikin, A. A. \& Sokoloff, D. D., 1983, Magnetic Fields in Astrophysics, Gordon and Breach, New York. 